\title{
Effectiveness of "Indocyanine Green Dye Clamp Test" in Selecting Perforators for Muscle-Sparing-2 Transverse Rectus Abdominis Myocutaneous Flaps in Breast Reconstruction
}

\author{
Seiko Okumura, $\mathrm{MD}^{1,2}$ Meisei Takeishi, MD, $\mathrm{PhD}^{3}$ \\ Ikuo Hyodo, MD ${ }^{1}$ Yuzuru Kamei, MD, $\mathrm{PhD}^{2}$ \\ ${ }^{1}$ Department of Plastic and Reconstructive Surgery, Aichi Cancer \\ Center Hospital, Nagoya, Aichi, Japan \\ 2 Department of Plastic and Reconstructive Surgery, Nagoya \\ University Graduate School of Medicine, Nagoya, Aichi, Japan \\ ${ }^{3}$ Breast Reconstruction Institute, Mishima, Japan
}

Ryota Nakamura, MD ${ }^{2}$ Satoshi Kakutani, MD ${ }^{1}$

Address for correspondence Seiko Okumura, MD, Department of Plastic and Reconstructive Surgery, Aichi Cancer Center Hospital, 1-1 Kanokoden, Chikusa-ku, Nagoya 464-8681, Aichi, Japan (e-mail: seiko-ok@tk9.so-net.ne.jp).

J Reconstr Microsurg Open 2017;2:e126-e131.

\begin{abstract}
Keywords

- MS2 TRAM flap

- breast reconstruction

- indocyanine green dye
\end{abstract}

Introduction In breast reconstruction for cases in which tissue from zones 1 to 4 is required or a lower midline scar exists, it is possible to predict the need for vascular anastomosis with a vascular pedicle on the contralateral side if the dominant territory of each perforator can be identified in advance. In addition, it is possible to determine whether a single- or multiple-perforator-based flap is needed. We, therefore, developed the "indocyanine green dye (ICG) clamp test" to select perforators for breast reconstruction.

Methods The blood flow of a perforator was blocked using a microvascular clamp when determining its inclusion in a flap. The clamps were released after the enlargement of the ICG fluorescence imaging range. The enlarged imaging range was then observed to determine whether an additional pedicle was required.

Subjects One hundred thirty-two breast reconstruction procedures had been performed using the free muscle-sparing- 2 transverse rectus abdominis myocutaneous (MS2 TRAM) flap method from May 2012 to December 2015. The population of the present study included 29 of these cases in which the selection of perforators was deemed necessary.

Results We investigated the cases in which anastomosis of the contralateral vascular pedicle was unnecessary. It was possible to preserve the medial muscle in $79.3 \%$ of the cases using our procedure.

Conclusion Our newly developed ICG clamp test was useful in selecting perforators for MS2 TRAM flaps and facilitated the performance of minimally invasive surgery. received

February 17, 2017

accepted after revision

July 31,2017
DOI https://doi.org/

10.1055/s-0037-1606360

ISSN 2377-0813.
Copyright $\odot 2017$ by Thieme Medical Publishers, Inc., 333 Seventh Avenue, New York, NY 10001, USA.

Tel: +1(212) 584-4662.
License terms

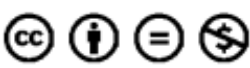


Perforator flaps within the rectus abdominis muscle are increasingly used in autologous breast reconstruction owing to recent developments in surgical techniques. However, due to individual differences in the vascular territory of a singleperforator-based flap, it is necessary to raise a bipedicle flap when tissue from zones 1 to 4 is required or when a lower midline scar is present. In addition, if the blood perfusion of the single-perforator-based flap is considered unreliable, a muscle-sparing-2 transverse rectus abdominis myocutaneous flap (MS2 TRAM flap), which is raised as a multipleperforator-based flap, is selected. The MS2 TRAM flap spares the muscles that are medial and lateral to the perforators. By identifying in advance the dominant territory of each perforator, it is possible to determine whether there is a need for vascular anastomosis with a vascular pedicle on the contralateral side, even in cases in which tissue from zones 1 to 4 is required, or in which a lower midline scar is present. It is also possible to determine whether a single- or multiple-perforator-based flap is required.

In the present study, we devised a method using indocyanine green dye (ICG) fluorescence angiography to help in determining the perforator territory that is required when raising the flap. The method is applied during surgery. The perforators and vascular pedicles are selected after the determination of the perfusion zone. Thus, the perforator rows and vascular pedicle on the contralateral side can be preserved in cases in which the vascular territory is extensive. In the present study, we examine cases in which this method was applied and investigate its effectiveness.

\section{Methods}

\section{Procedure I}

For cases in which a lower midline scars is present or when tissue from zones 1 to 4 of the vascular pedicle on the contralateral side is required and in which vascular anastomosis is deemed necessary, the following procedure is performed.

Prior to surgery, the position of the perforators and the pedicle course are identified using contrast-enhanced computed tomography (CT) and are marked on the abdominal region. The flap is designed in the standard position for a TRAM flap. The superior margin of the flap is located at one fingerbreadth superior to the umbilical fossa. After making an incision on the superficial fascia, the deep subcutaneous adipose tissue beneath the superficial fascia is collected on the transverse axis of the flap $(\sim 5 \mathrm{~cm}$ longer than the skin incision), along with part of the deep subcutaneous adipose tissue. The flap forms a rectangle. The flap is then raised from the surface of the fascia, opposite the primary vascular pedicle. Once the perforators are identified and preserved to avoid damage ( $\mathbf{- F i g . 1}$ ), the other parts are elevated from the surface of the fascia to reach the midline. On the side of the primary vascular pedicle, the flap is raised from the lateral side. The lateral row perforators are identified, and the anterior sheath of the rectus abdominis muscle-through which the perforator penetrates-is opened and cut along the lateral side of the perforators. The perforators are dissected toward the centrum to reach the deep inferior epigastric vessels. Dissection is continued along the surface of the fascia. The medial row perforators are identified, and the circumference of the perforators is dissected from the surface of the fascia. Thus, only the perforators remain connected to the fascia. The umbilicus is then hollowed out and was preserved (-Fig. 2 ).

The primary perforators are left open, while the blood flow of the perforators on the contralateral side (with which vascular anastomosis is implemented) is blocked using a microvascular clamp (clamp). ICG (2 cc; $2.5 \mathrm{mg} / \mathrm{mL}$, intravenous and systemic) is administered. After the administration of ICG, the imaging range, which is observed using a HyperEye Medical System (Mizuho) or Photodynamic Eye (Hamamatsu Photonics), begins to grow (-Fig. 3). The clamp is released when the imaging range has been identified, after the enlargement of the imaging range stops. In cases in which the vascular pedicle is located on one side, if the imaging range is sufficiently large, and the required tissue mass is considered to be implantable, vascular anastomosis of the contralateral side is unnecessary. If the tissue mass is deemed insufficient (based on the imaging range), in such cases, vascular anastomosis is performed with the vascular pedicle on the contralateral side (-Fig. 4).

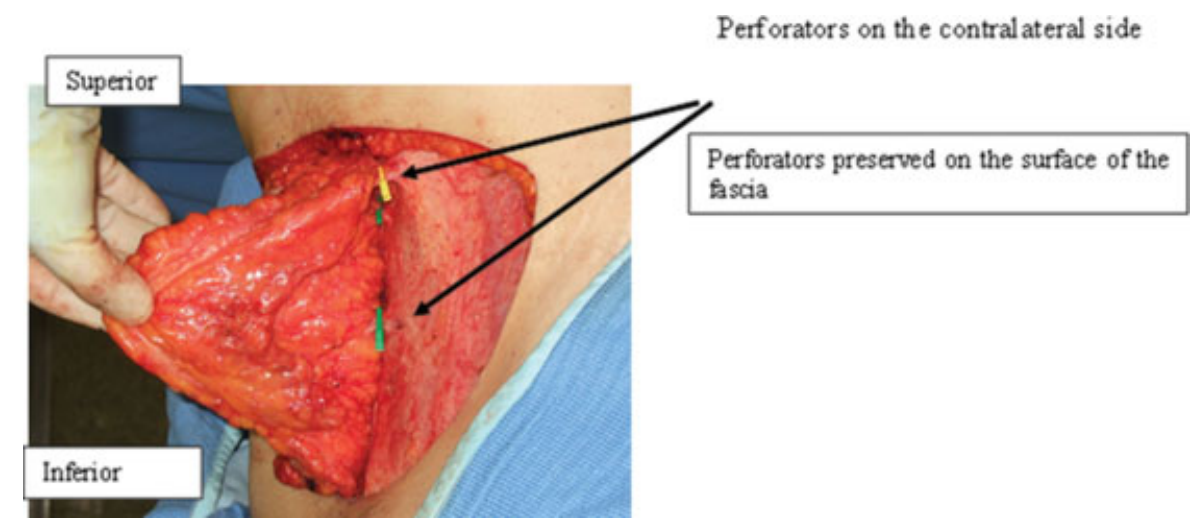

Fig. 1 Dissecting from the surface of the fascia, leaving the perforators behind on the contralateral side. Arrows: Perforators preserved on the surface of the fascia. 


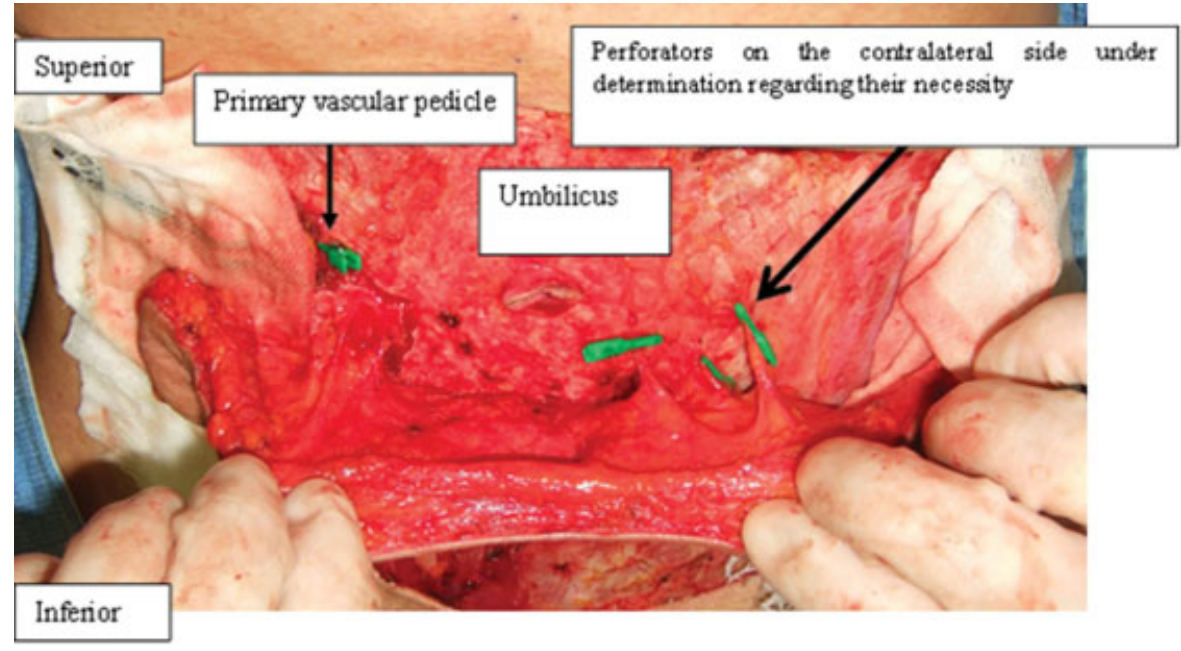

Fig. 2 Only the perforators connected with the surface of the fascia. The circumference of perforators is dissected from the surface of the fascia, leaving the perforators behind. The umbilicus is hollowed out, leaving only the perforators connected with the fascia.

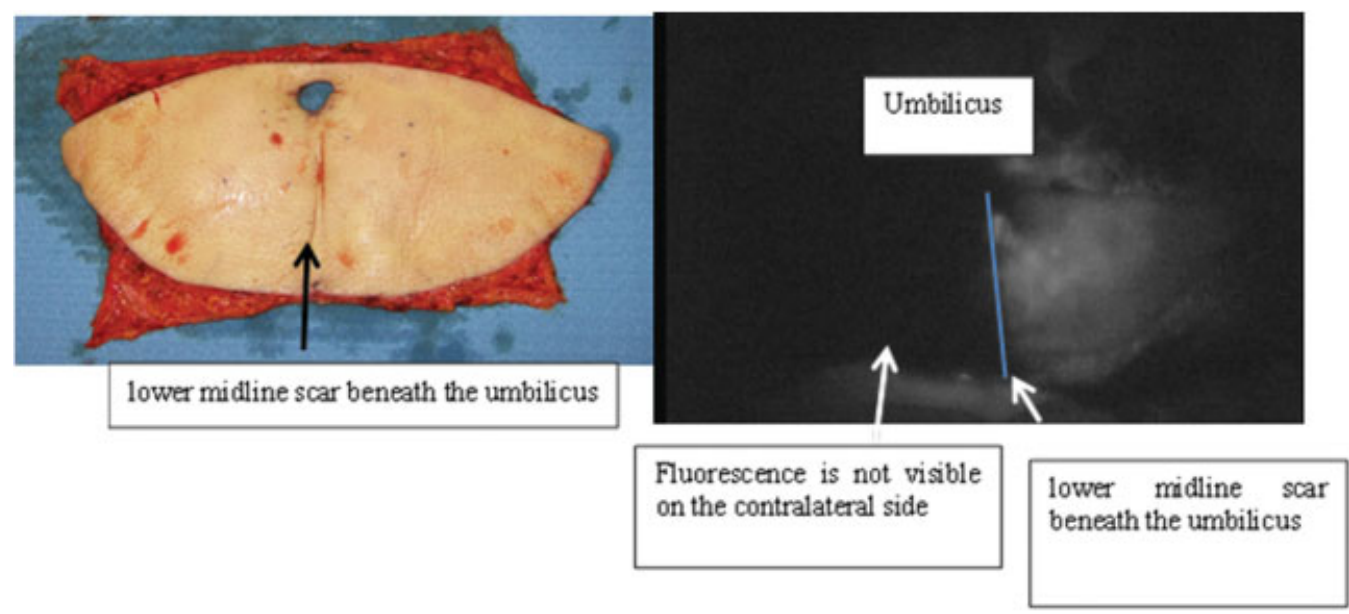

Fig. 3 A case in which the lower midline scar exists (upon angiography with the perforators on the contralateral side clamped). The vascular pedicle is on the left side and the perforators on the right side are clamped upon ICG fluorescence angiography. The contrast medium is applied, without going beyond the midline, on the right side of the blue line (contralateral side). The addition of vascular anastomosis is deemed necessary.
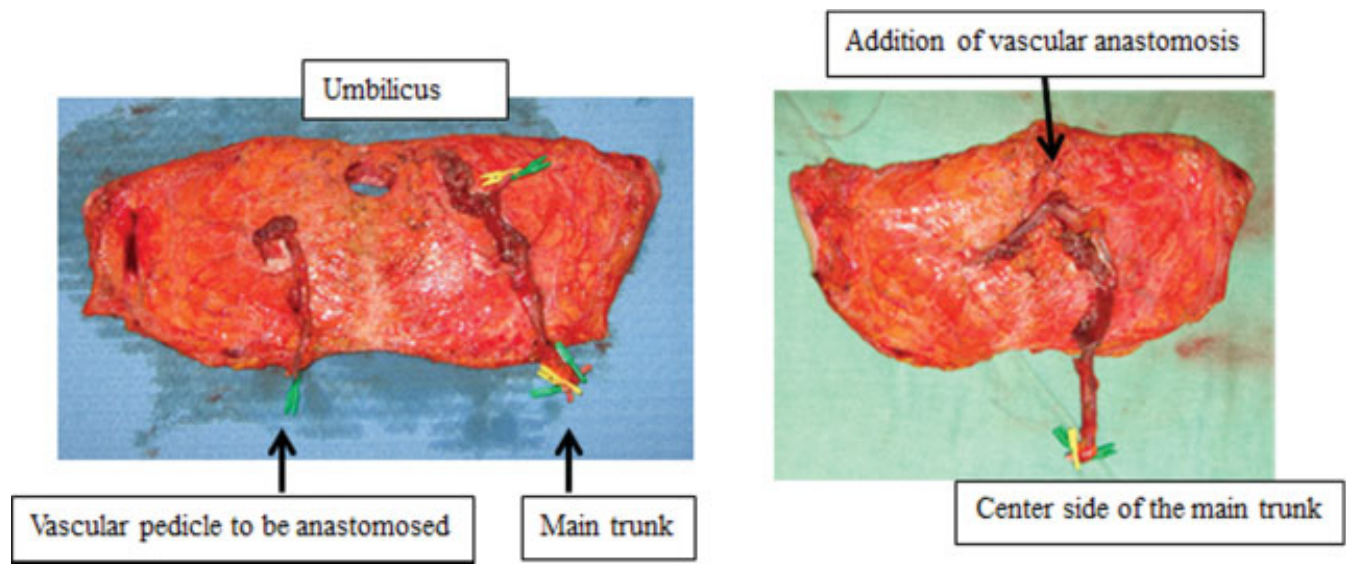

Fig. 4 Addition of vascular anastomosis. On the peripheral side of the main trunk, the central side of the vascular pedicle to be anastomosed is anastomosed within the skin flap, end-to-end, with both arteries and veins. 


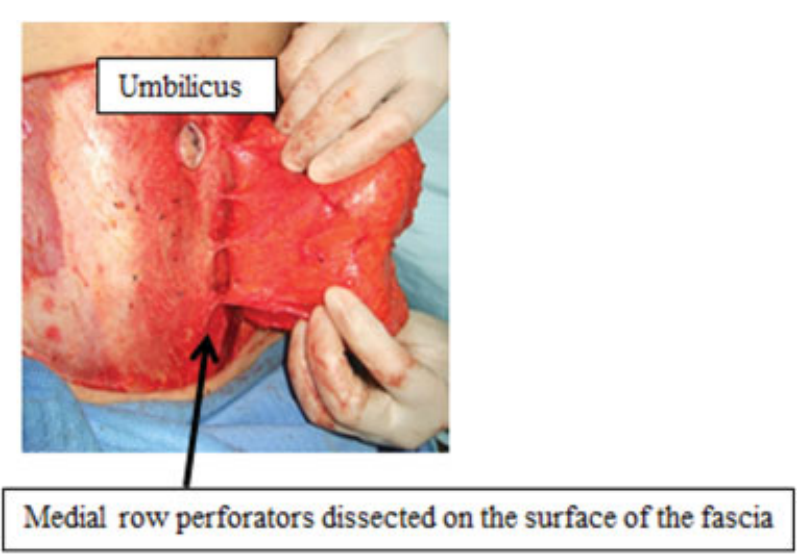

Fig. 5 The medial row perforators dissected on the surface of the fascia The medial row perforators on the side of the primary vascular pedicle are identified and the circumference of the perforators is dissected from the surface of the fascia, leaving the perforators behind.

\section{Procedure II}

In cases treated using an MS2 TRAM flap or a DIEP flap, the flap design and skin incision are performed according to the procedure described in I, and the same abdominal wall approach is applied. The lateral row perforators are dissected, similarly as described in procedure I. These lateral row perforators are regarded as the primary perforators. The flap on the contralateral side of the primary vascular pedicle is raised from the surface of the fascia. The perforators on the contralateral side are all ligated, and the flap on the fascia is dissected to reach the midline. The umbilicus is hollowed out beyond the midline. The medial row perforators on the side of the primary vascular pedicle are then identified, and the circumference of the perforators is dissected from the surface of the fascia, leaving the perforators behind (-Fig. 5 ).

The primary lateral row perforators are left open, while the medial row perforators are clamped. ICG is administered. After the enlargement stops, the imaging range is recorded on the skin flap, and the clamp is released (-Fig. 6).

If the imaging range is barely enlarged, and it is determined that the necessary tissue mass can be implanted using the lateral row perforators alone, the medial row perforators are

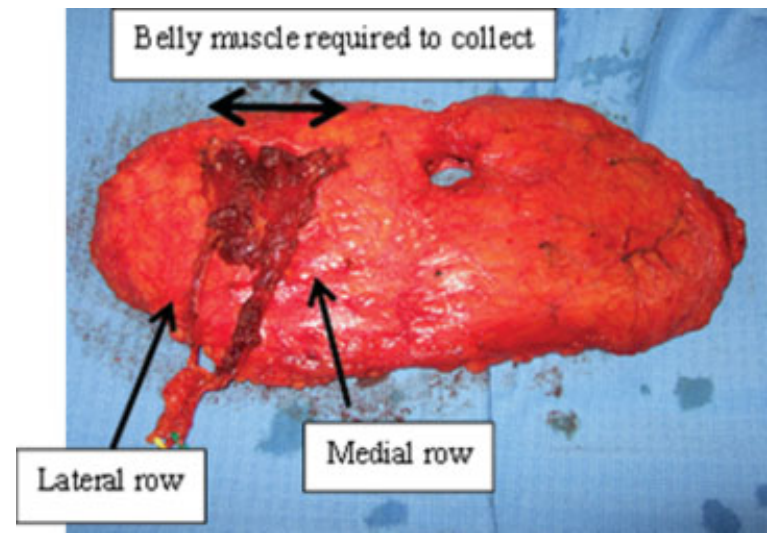

Fig. 7 The raised flap. The width of collected muscle was enlarged since the raised flap included the medial row.

ligated, and the flap is raised using the lateral row perforators alone. If the imaging range is significantly enlarged after the release of the clamp, and it is determined that tissue mass in the enlarged range is required, the flap is raised with both the lateral and medial row perforators (-Fig. 7).

\section{Subjects}

One hundred thirty-two breast reconstruction procedures (immediate reconstruction, $n=103$; delayed reconstruction, $n=29$ ) had been performed using the free MS2 TRAM flap method from May 2012 to December 2015. The population of the present study included 29 of these cases in which the selection of perforators was deemed necessary. Specifically, these were cases in which patients had lower midline scars $(n=11)$; cases in which it was deemed necessary to extend the flap beyond the zone II/IV boundary based on the measurement of the chest skin length from the clavicle to the inframammary fold (IMF) $(n=13)$ ( - Fig. 8); and cases in which it was deemed necessary to extend the flap based on preoperative contrast-enhanced CT findings showing that the distance between the part raised from the medial row perforator muscle and the part raised from the lateral row perforators muscle would exceed two-thirds of
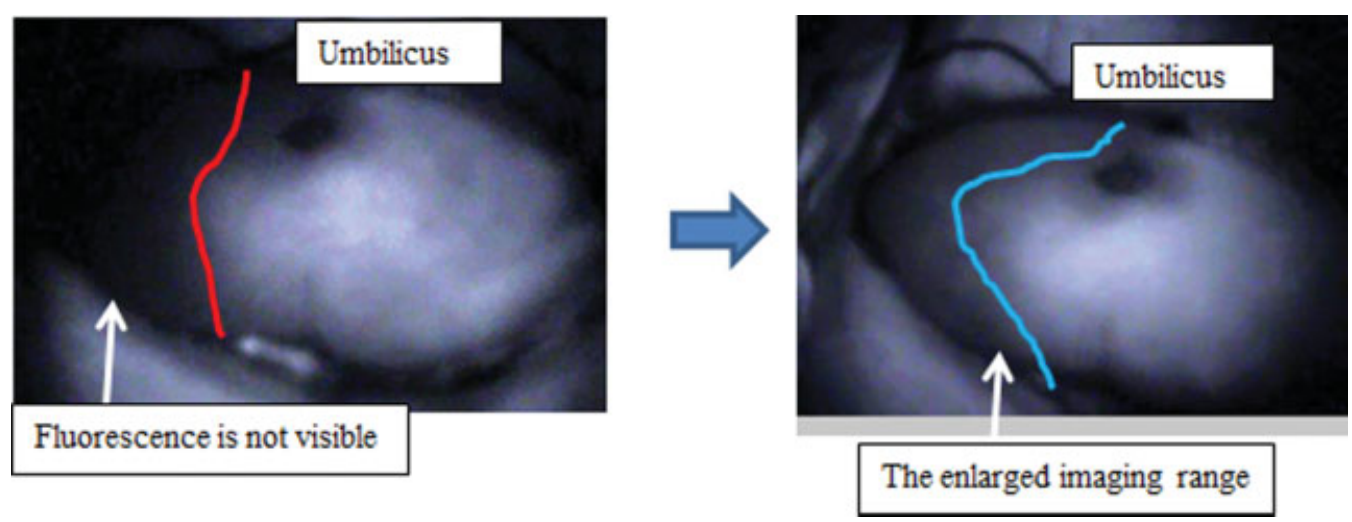

Fig. 6 Angiography is conducted with the blood flow of the medial row perforators blocked, and the imaging range was enlarged after the release of the clamps. Left: The left side of the red line is the imaging range with the medial row perforators clamped. Right: The imaging range was enlarged to the blue line when the medial row was included. 


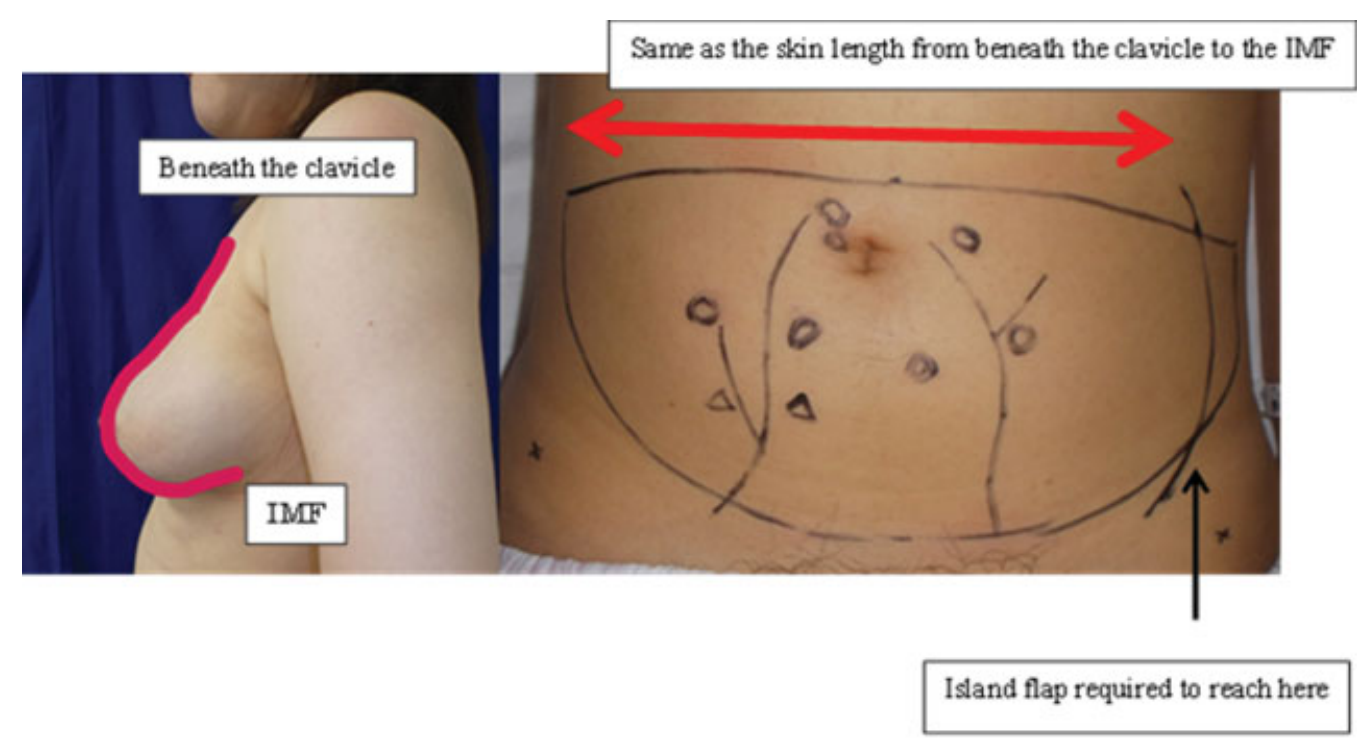

Fig. 8 The design prior to surgery. Left: The red line shows the skin length from beneath the clavicle to the IMF. Right: $\leftarrow \rightarrow$ has the same length from the clavicle to the IMF.

the length of the entire muscle, indicating a significant sacrifice of muscle $(n=5)$.

\section{Results}

In 13 cases, a great deal of tissue mass was required, and it was necessary to determine whether vascular anastomosis to the vascular pedicle on the contralateral side was required. Vascular anastomosis was ultimately implemented in two (15.4\%) of these cases. A lower midline scar existed in 11 cases, and vascular anastomosis was ultimately implemented in two $(18.2 \%)$ of these cases.

In five cases, the surgeons had to choose between a DIEP flap or an MS2 TRAM flap. This included two (40\%) cases in which medial row perforators were considered to be required, and in which an MS2 TRAM flap was raised.

In $79.3 \%(23 / 29)$ of the cases, the clamping of perforators during ICG fluorescence angiography was deemed unnecessary, making it possible to preserve the vascular pedicle on the contralateral side and to preserve the muscle medial to the lateral row perforators.

The flaps survived in all cases. There was no consolidation due to necrosis of a partial flap or fat necrosis in any of the cases. Hernia/bulging occurred in five cases. It was impossible to preserve the medial row perforators in two of these five cases.

\section{Discussion}

A previous study reported that the imaging range of ICG fluorescence angiography was almost the same as the surviving area of the flap in cases that involved pedicled TRAM flap procedures. Another study evaluated the rate of fat necrosis within the flap after surgery in cases that were performed before ICG fluorescence angiography and in cases in which only the imaging range on ICG fluorescence angiography was investigated. The study revealed that the rate of fat necrosis was lower in cases in which the range was determined using angiography. The findings suggested that angiography was effective and highly reliable for determining the area in which the flap can survive. ${ }^{1,2}$ In the present study, no necrosis (including fat necrosis) occurred within the imaging range obtained by ICG fluorescence angiography. This result was considered to be highly reliable.

To facilitate the safe elevation of a flap, contrast-enhanced CT generally is performed prior to surgery to clarify the perforators, that are to be included, and the pedicle course. ${ }^{3,4}$ However, contrast-enhanced CT cannot determine the dominant territory of each perforator. Reticular choke vessels are located on the periphery of the perforators, where adjacent choke vessels anastomose and communicate with each other (choke anastomosis). According to the angiosome theory, while the blood flow from the primary vascular territory of a vascular pedicle can extend its territory to the second vascular territory, and beyond the first site of choke anastomosis, it cannot extend beyond the second site of choke anastomosis. ${ }^{5,6}$

The range in which a flap can survive is determined based on two factors: the alignment of perforators of the vascular pedicle on the contralateral side and the degree of choke anastomosis between the perforators of the two vascular pedicles. Preoperative contrast-enhanced CT can determine the pedicle course within the muscle and the arrangement of the perforators, but cannot predict choke anastomosis, which defines the vascular territory of the flap. This can effectively be determined by ICG fluorescence angiography.

The "ICG clamp test," allows the vascular territory of the perforators, which are thought to meet the minimum requirements, to be determined before the blood vessels are raised from inside the muscle. Thus, it allows the surgeon to determine whether the addition of a vascular pedicle on the contralateral side or the medial row perforators is needed. Consequently, the blood vessels that are not required for flap survival can be preserved. In the present study, in $79.3 \%$ of cases in which the addition of a vascular pedicle was initially thought to be required, the addition was ultimately deemed 
to be unnecessary-indicating the utility of this method for intraoperative decision making.

If lateral and medial row perforators exist, they, respectively, have distinct dominant territories. It has been reported that lateral and medial row perforators strongly dominate zones 3 and 2, respectively. The MS2 TRAM flap, which includes both the lateral and medial row if possible, is thought to be more effective than the DIEP flap in regard to avoiding partial necrosis. Based on this test, although partial necrosis and fat necrosis did not occur in DIEP flaps determined as having satisfactory blood perfusion, our results revealed that only an MS2 TRAM flap could be implanted with good blood perfusion in $40 \%$ of all cases.

It has been reported that, regarding complications associated with flaps, such as necrosis/partial necrosis of flaps and fat necrosis, fat necrosis following surgery occurs at a lower rate with multiple-perforator-based flaps raised rather than with single-perforator-based flaps. Moreover, the incidence rate thereof with DIEP flaps is reported to be significantly higher than that with conventional TRAM flaps. Since the hemodynamics of MS2 TRAM flaps, which include both lateral and medial row perforators, is thought to be nearly the same as that of conventional TRAM flaps, MS2 TRAM flaps are more effective in reducing complications associated with flaps than DIEP flaps. Therefore, we, basically, elevate the MS2 TRAM flap as the first choice. This method is also thought to be effective as it enables an appropriate determination to be made between either a DIEP flap or an MS2 TRAM flap.

Our elevated MS2 TRAM flap contains both lateral and medial row perforators as confirmed by $\mathrm{CT}$, generally including three to seven perforators. By separating the muscles from the perforators and exfoliating only the blood vessels, we try to preserve the muscles.

In our method, we primarily want to elevate the MS2 TRAM flap. To achieve this, we first secure the lateral row perforators and carefully check the main duct of the vascular pedicle. Consequently, we believe that it is not possible to use the medial row as the basic axis. While in some cases the medial row perforators are the dominant vessels (having dominant territories), the lateral row perforators must be the basic axis in our method.

Hernia and/or abdominal distension is a noteworthy complication of abdominal surgery. Conventional TRAM flaps have been reported to be associated with a higher incidence of these complications in comparison to DIEP flaps; ${ }^{7-11}$ however, the incidence of hernia/abdominal distension did not differ to a statistically significant extent between patients who received MS2 TRAM and those who received DIEP flaps. ${ }^{7-10}$ Furthermore, the incidence of hernia/abdominal distension with MS2 TRAM flaps is equivalent to the average incidence of hernia/abdominal distension in patients who receive conventional TRAM and DIEP flaps. ${ }^{7,11}$

If it is possible to implant the tissue from zones 1 to 4 using the lateral row alone, the flap should be raised using the lateral row alone. In addition, to avoid abdominal complications, if the distance between the lateral and medial rows is sufficiently long, surgeons should check to see if the lateral row alone is sufficient, as this can prevent unnecessary muscle sacrifice.

The incidence of hernia/abdominal distension in cases involving bilateral vascular pedicle collection is reported to be higher than that in cases involving unilateral collection. ${ }^{11,12}$ Furthermore, the incidence of hernia is reported to be higher in patients with lower midline scars. ${ }^{12}$ In cases in which the unilateral vascular pedicle can be transplanted with tissue from zones 1 to 4 , the incidence of postoperative hernia can be reduced to a greater degree in comparison to cases in which bilateral vascular pedicles are used. Our clamp test is thought to be effective for minimizing muscle sacrifice.

\section{Conclusion}

The elevation of a bipedicle flap for vascular anastomosis and the elevation of a flap that includes both the medial and lateral row perforators increases abdominal invasion. Our novel method, which identified the perforators that were required for survival, was thought to be effective for minimizing this invasion and avoiding unnecessary surgery.

\section{References}

1 Yamaguchi S, De Lorenzi F, Petit JY, et al. The "perfusion map" of the unipedicled TRAM flap to reduce postoperative partial necrosis. Ann Plast Surg 2004;53(03):205-209

2 Holm C, Mayr M, Höfter E, Becker A, Pfeiffer UJ, Mühlbauer W. Intraoperative evaluation of skin-flap viability using laser-induced fluorescence of indocyanine green. Br J Plast Surg 2002; 55(08):635-644

3 Rozen WM, Palmer KP, Suami H, et al. The DIEA branching pattern and its relationship to perforators: the importance of preoperative computed tomographic angiography for DIEA perforator flaps. Plast Reconstr Surg 2008;121(02):367-373

4 Tong WM, Dixon R, Ekis H, Halvorson EG. The impact of preoperative CT angiography on breast reconstruction with abdominal perforator flaps. Ann Plast Surg 2012;68(05):525-530

5 Taylor GI, Palmer JH. The vascular territories (angiosomes) of the body: experimental study and clinical applications. Br J Plast Surg 1987;40(02):113-141

6 Cormack GC, Lamberty BGH. The Arterial Anatomy of the Skin Flap. Edinburgh: Churchill Livingstone; 1986:1-13

7 Man LX, Selber JC, Serletti JM. Abdominal wall following free TRAM or DIEP flap reconstruction: a meta-analysis and critical review. Plast Reconstr Surg 2009;124(03):752-764

8 Nahabedian MY, Tsangaris T, Momen B. Breast reconstruction with the DIEP flap or the muscle-sparing (MS-2) free TRAM flap: is there a difference? Plast Reconstr Surg 2005;115(02):436-444, discussion 445-446

9 Nelson JA, Guo Y, Sonnad SS, et al. A Comparison between DIEP and muscle-sparing free TRAM flaps in breast reconstruction: a single surgeon's recent experience. Plast Reconstr Surg 2010; 126(05):1428-1435

10 Vyas RM, Dickinson BP, Fastekjian JH, Watson JP, Dalio AL, Crisera CA. Risk factors for abdominal donor-site morbidity in free flap breast reconstruction. Plast Reconstr Surg 2008;121(05):1519-1526

11 Wan DC, Tseng CY, Anderson-Dam J, Dalio AL, Crisera CA, Festekjian JH. Inclusion of mesh in donor-site repair of free TRAM and muscle-sparing free TRAM flaps yields rates of abdominal complications comparable to those of DIEP flap reconstruction. Plast Reconstr Surg 2010;126(02):367-374

12 Takeishi M, Shaw WW, Ahn CY, Borud LJ. TRAM flaps in patients with abdominal scars. Plast Reconstr Surg 1997;99(03):713-722 